\title{
Differing Effect of an EMG Biofeedback-Monitored Exercise Compared to an Un-monitored One in Vastus Medialis Obliquus and Vastus Lateralis Muscles Amplitude in Knee Osteoarthritis
}

\author{
Umi Syayyirotin A, Lukitra Wardhani, Fatchur Rochman \\ Department of Physical Medicine and Rehabilitation Faculty of Medicine Airlangga University - Dr. \\ Soetomo Hospital, Surabaya - Indonesia
}

\begin{abstract}
Objectives: The objective of the study was to know the difference of maximum and ratio amplitudes of vastus medialis obliquus and vastus lateralis muscles before and after exercise with and without EMG biofeedback monitoring in knee osteoarthritis patients.

Methods: Randomized clinical study design. This study was conducted in Department of Physical Medicine and Rehabilitation Airlangga University, Dr. Soetomo hospital Surabaya. Twenty eight patients with knee osteoarthritis were randomly placed into 2 groups, the first group received progressive resistive exercise with EMG biofeedback, while the other group received training with progressive resistive method only. The ratio and maximum amplitudes of vastus medialis obliquus and vastus lateralis muscles were analyzed before and after 8 weeks exercise.

Results: The amplitudes of vastus medialis obliquus and vastus lateralis were improved significantly in group receiving progressive resistive training method with EMG biofeedback monitoring $(\mathrm{p}=.01$ and $\mathrm{p}=.001$, respectively). Comparison between two groups after 8 weeks of progressive resistive exercise showed significant improvements on amplitude of vastus medialis obliquus muscle $(\mathrm{p}=.014)$ and amplitude ratio of $\mathrm{VMO} / \mathrm{VL}(\mathrm{p}=.034)$ in EMG biofeedback monitoring group.

Conclusions: Progressive resistive exercise with EMG biofeedback monitoring could improve significantly the amplitudes of vastus medialis obliquus and vastus lateralis muscles and the amplitude ratio of $\mathrm{VMO} / \mathrm{VL}$ in knee osteoarthritis patients.
\end{abstract}

Keywords: knee osteoarthritis, progressive resistive exercise, EMG biofeedback monitoring.

\section{INTRODUCTION}

Osteoarthritis $(\mathrm{OA})$ is a degenerative disease of joint cartilage which frequently happens. This disease is affected by genetic factor, aging, metabolism, and joint movement activity. ${ }^{1}$ Muscles in the knee joint are divided into 2 groups, knee extensor and knee flexor. Main

Received in March 2012 and accepted for published in April 2012.

Correspondence address : Umi Syayirotin, Email : umisyayirotin@yahoo.com knee extensor is femoral quadriceps which inserts in tibia and patella that consist of 3 layers, i.e. superficial layer (rectus femoris muscle), middle layer (vastus lateralis and medialis vastus muscles), and deep layer (vastus intermedius muscle). All knee extensor tendons insert in tibial tuberosity through the anterior part above patella.,3 Medial vastus muscle consist of 2 groups of muscle fibers with different direction. A more distal muscle fiber (vastus medialis obliquus) directed to patella on the angle $50-55^{\circ}$, while the more longitudinal muscle fiber (vastus medialis longus) directed to patella on the angle $15-18^{\circ}$, due to the 
condition these muscle fibers have different line of force to patella. Cross sectional area of vastus medialis obliquus muscle is only $30 \%$ of the whole vastus medialis muscle, nevertheless the oblique stretch of this muscle has important role to stabilisation and orientation of patella when moving around the femoral intercondyle sulcus. $^{2}$

Patella has important biomechanical role that aid the quadriceps muscle to extend by elongation of lever arm and enable better compression stress distribution of the femur. When the quadriceps muscle contracts, vastus medialis obliquus (VMO) muscle plays a role to pull the patella to medial and keep patella in the femoral sulcus. Vastus medialis muscle plays a role in $10 \mathrm{o}-15 \mathrm{o}$ of end extension. ${ }^{3}$

Changes in OA are unven surface of joint cartilage followed by ulceration and loss of joint cartilage that enable contact between bone and bone within the joint, then forming subchondral cyst, osteophytes at the bone margins and inflammation reaction of synovial membrane. Enlargement of joint, thickening of synovial membrane and joint capsule, and ligament stretch causes instability and deformity. ${ }^{4}$ Muscles around the joint are weakened due to synovial effusion and disuse atrophy of unilateral side and muscle spasm of the contralateral side. ${ }^{5}$

Progressive resistive exercise (PRE) is a form of dynamic isotonic strengthening exercise with gradually increasing load. Strengthening exercise with PRE is better in maintaining and improving muscle function, reduce joint pain, and increase function of knee OA patients. ${ }^{67}$ Biofeedback is a technique that use electronical device which shows internal physiological process in human body, either in normal or abnormal, in the form of visual and audio signals as direction to manipulate voluntary process through signal manipulation shown in biofeedback machine. ${ }^{8,9}$ Electromyographic (EMG) biofeedback is a media that gives feedback in the audio visual signal, in which non-functional or inadequate proprioceptor system can be replaced by visual and auditory systems. ${ }^{8,9}$

The role of EMG biofeedback monitoring on the amplitude ratio of VMO and VL in OA patients that received exercise with PRE method is not clear. This study aims to compare the amplitude ratio of VMO and VL before and after exercise of PRE method with and without EMG biofeedback monitoring in knee OA patients.

Hypothesis of this study were there was difference of amplitude ratio of vastus medial obliquus and vastus lateral muscles between before and after exercise of progressive resistive exercise method with or without EMG biofeedback monitoring in knee osteoarthritis patients.

There was also difference of amplitude ratio of vastus medial obliquus and vastus lateral muscles before and after exercise of progressive resistive exercise method between EMG biofeedback monitoring and without EMG biofeedback monitoring in knee osteoarthritis patients.

\section{METHODS}

This study was a clinical trial, a simple randomization was taken which distinguished 2 groups. The first group received PRE exercise with EMG Biofeedback monitoring and the second group received PRE exercise without EMG Biofeedback monitoring. Comparative analysis was done on the ratio amplitude and maximum amplitude of the vastus medialis obliquus (VMO) and vastus lateralis (VL) muscles before and after exercise for 8 weeks. Inclusion criterias were knee OA patients aged 50-60 years, BMI 25-30, normal knee ROM, radiological results show knee OA with grade 1 and 2 according to Kellgren \& Lawrence, the muscle strength of quadriceps is more than 3, pain scale (visual analog scale/VAS) 3-6. Patients will be excluded if she has one or more exclusion criterias as follows: has trauma or fracture of lower extremity and knee injury of the previous 6 months, has neurological impairment, muscle disease, is not cooperative, has moderate to severe hypertension.

Amplitude of VMO and VL muscles was the amplitude of electrical activity generated by the muscle contraction of VMO and VL muscles. In this study, maximal amplitude was measured in microvolt by EMG biofeedback machine type Myomed 932. The amplitude was measured from the baseline when firing 
pattern to the top of maximal wave formed when contraction in the period of work time. Evaluation of the initial and amplitude of end exercise with PRE method were measured from the mean maximal amplitude within three times of measurements. The exercise with the technique progressive resistive exercise (PRE) was the dynamic strengthening exercise with gradually increased load that the muscle contract consentrically along with the range of joint motion. The exercise procedures of the strengthening exercise with the progressive resistive exercise (PRE) technique was implemented in the following manners: a) determining the $10 \mathrm{RM}$ load with a sand bag, b) implementing the 10 repetitions exercise with the load $1 / 2$ of the $10 \mathrm{RM}, \mathrm{c}$ ) implementing the 10 repetitions exercise with the load $3 / 4$ of the 10 RM. d) implementing the 10 repetitions exercise with the full load of the $10 \mathrm{RM}$, e) Subject take a brief rest (2-5 minutes) between the exercise bouts, motion was carried on the whole joint range of motion, hold 6 seconds at the end of joint range of motion, then put the leg down and subject take 5 seconds rest before starting the next repetitions, f) Evaluation of 10 $\mathrm{RM}$ was done every week to determine the next exercise load.

Exercise was done once daily for 5 days in a week, 2 times per week in outpatient clinic of Medical Rehabilitation Installation of RSUD Dr.Soetomo under supervision of the researcher and assistance of trained physiotherapist. The other 3 days of exercises were done at home by the subjects themselves with determined load under phone call supervision.

The collected data were the results of amplitude of the VMO and VL muscles, the ratio of $\mathrm{VMO} / \mathrm{VL}$, and subjects compliance. Collected data was analyzed descriptively to normalized data by Kolmogorov-Smirnov test previously, followed by inferentialy analayzing.

\section{RESULTS}

Total study subjects were 28 subjects divided into 2 groups, 14 subjects was in the group 1 in which the exercise method of Progressive resistive exercise (PRE) was done with EMG Biofeedback monitoring and 14 subjects were included in group 2 in which the exercise method of Progressive resistive exercise (PRE) was done without EMG Biofeedback monitoring. The study subjects distibution consisted of 25 females and 3 males.

Data normality test was done to the study results by the statistical test Kolmogorov-Smirnov. Analysis by paired $t$ test was done to normal data distribution while Wilcoxon Signed Ranks test was done to data with not normal distribution to compare the difference of values before and after exercise program in each group. To obtain a significant difference between 2 groups was analyzed by t-independent sample on normal distribution data and Mann-Whitney test analysis in data which was not normally distributed. Significance $\mathrm{p}$-value used was $\mathrm{p}<0.05$.

Table 1. Data normality test of exercise group with EMG Biofeedback Monitoring

\begin{tabular}{lllll}
\hline & Mean & SD & $p$ & Significance \\
\hline Age & 56.36 & 2.82 & 0.065 & Not significant \\
BMI & 27.25 & 1.63 & 0.200 & Not significant \\
Attendance of exercise & 19.50 & 0.86 & 0.0001 & Significant \\
Amplitude of initial VMO & 78.17 & 43.72 & 0.200 & Not significant \\
Amplitude of initial VL & 112.33 & 42.03 & 0.160 & Not significant \\
Amplitude of end VMO & 120.45 & 47.26 & 0.200 & Not significant \\
Amplitude of end VL & 112.33 & 42.03 & 0.001 & Significant \\
Amplitude ratio of initial VMO/VL & 0.64 & 0.22 & 0.200 & Not significant \\
Amplitude ratio of end VMO/VL & 0.77 & 0.21 & 0.185 & Not significant \\
\hline
\end{tabular}


Table 1 shows the result of data normality test in PRE method exercise group with EMG biofeedback monitoring. According to normality test there was not a normal distribution on initial VMO and end VL amplitude according to $p$ value obtain was $<0.005$.

Table 2. Data normality test of exercise group without EMG Biofeedback Monitoring

\begin{tabular}{lllll}
\hline & Mean & SD & $\mathrm{p}$ & Significance \\
\hline Age & 56.15 & 3.46 & 0.200 & Not significant \\
BMI & 28.42 & 1.99 & 0.09 & Significant \\
Attendance of exercise & 19.38 & 0.96 & 0.0001 & Significant \\
Amplitude of initial VMO & 65.97 & 64.55 & 0.001 & Significant \\
Amplitude of initial VL & 101.00 & 64.56 & 0.059 & Not significant \\
Amplitude of end VMO & 78.30 & 40.55 & 0.200 & Not significant \\
Amplitude of end VL & 130.72 & 49.51 & 0.078 & Not significant \\
Amplitude ratio of initial VMO/VL & 0.601 & 0.29 & 0.200 & Not significant \\
Amplitude ratio of end VMO/VL & 0.602 & 0.21 & 0.200 & Not significant \\
\hline
\end{tabular}

Table 2 showed the result of data normality rest of PRE method exercise group without EMG biofeedback monitoring. From the results of mean age, amplitude of end VMO muscle, initial and amplitude of end VL muscle, amplitude ratio of $\mathrm{VMO} / \mathrm{VL}$ initial and end exercise, initial and end amplitude ratio of $\mathrm{VMO} / \mathrm{VL}$ and amplitude ratio of VMO/VL p-value $>0.05$ showed data with normal distribution. From mean BMI, exercise attendance and amplitude of initial VMO, p-value $<0.05$ showed data with not normal distribution.

Data normality for sex distribution between PRE method exercise group with EMGBiofeedback monitoring and without EMG Biofeedback monitoring use the Chi Square statistical test with the $p$-value $=1.00(p>0.05)$ which showed normal data distribution in both groups.

Data analysis in each group with normal distribution was done with paired t-test and for data with not normal distribution was done with Wilcoxon Signed Ranks test.

Table 3. Exercise group with EMG Biofeedback Monitoring

\begin{tabular}{cccccccc}
\hline & \multicolumn{2}{c}{ Before exercise } & \multicolumn{2}{c}{ After exercise } & \multirow{2}{*}{ Signification } \\
\cline { 2 - 5 } & Mean & SD & Mean & SD & & & Significant \\
\hline Amplitude of VMO & 78.17 & 43.72 & 120.45 & 47.26 & & 0.01 & Significant \\
Amplitude of VL & 112.33 & 42.03 & 155.34 & 38.69 & & 0.001 & Not significant \\
\hline
\end{tabular}

Table 3 showed study results of mean amplitude of VMO, VL muscles and amplitude ratio of $\mathrm{VMO} / \mathrm{VL}$ muscle before and after exercise PRE method with EMG biofeedback monitoring. Mean amplitude of VMO muscle before exercise was $78.17 \mu \mathrm{V}$, after exercise it became $120.45 \mu \mathrm{V}$. Mean amplitude of VL muscle was $112.33 \mu \mathrm{V}$ before exercise and was $155.34 \mu \mathrm{V}$ after exercise. Mean amplitude ratio of VMO/VL muscles before exercise was 0.64 and after exercise was 0.77 . When calculated with statistical analysis the mean amplitude of VMO muscle increased significantly after exercise with $\mathrm{p}$-value $=0.001$. The mean amplitude of VL muscle increased significantly after exercise with $\mathrm{p}$-value $=0.0001$. The mean amplitude ratio of VMO/VL muscles increased not significantly after exercise with $\mathrm{p}=0.183$. 
Table 4. Exercise group without EMG Biofeedback Monitoring

\begin{tabular}{cccccccc}
\hline & \multicolumn{2}{c}{ Before exercise } & \multicolumn{2}{c}{ After exercise } & \multirow{2}{*}{ Signification } \\
\cline { 2 - 5 } & Mean & SD & Mean & SD & & Not significant \\
\hline Amplitude of VMO & 65.97 & 64.55 & 78.31 & 39.18 & & 0.074 & \\
Amplitude of VL & 99.36 & 58.51 & 129.86 & 47.68 & 0.055 & Not significant \\
Amplitude ratio of VMO/VL & 0.59 & 0.29 & 0.60 & 0.20 & 0.940 & Not significant \\
\hline
\end{tabular}

Table 4 shows study results of mean amplitude of VMO, VL muscles and amplitude ratio of $\mathrm{VMO} / \mathrm{VL}$ muscle before and after exercise PRE method without EMG biofeedback monitoring. Mean amplitude of VMO muscle before exercise was $65.97 \mu \mathrm{V}$, after exercise it became $78.31 \mu \mathrm{V}$. Mean amplitude of VL muscle was $99.36 \mu \mathrm{V}$ before exercise and was $129.86 \mu \mathrm{V}$ after exercise. Mean amplitude ratio of $\mathrm{VMO} / \mathrm{VL}$ muscles before exercise was 0.59 and after exercise was 0.60 . Statistical analysis of the mean amplitude of VMO muscle show no significant improvement after exercise $(\mathrm{p}=$ 0.074). The mean amplitude of VL muscle did not increase significantly after exercise $(p=$ 0.055). The mean amplitude ratio of $\mathrm{VMO} /$ VL muscles did not increase significantly after exercise $(p=0.940)$.

Analysis of data between groups in normal distribution data was done by the t-independent sample test while for data with not normal distribution, test was done by the MannWhitney test.

Table 5. Differences between two exercise groups with and without EMG Biofeedback Monitoring

\begin{tabular}{cccccccc}
\hline & \multicolumn{2}{c}{$\begin{array}{c}\text { Group with } \\
\text { EMG biofeedback } \\
\text { monitoring }\end{array}$} & \multicolumn{2}{c}{$\begin{array}{c}\text { Group without } \\
\text { EMG biofeedback } \\
\text { monitoring }\end{array}$} & $p$ & Signification \\
\cline { 2 - 5 } & Mean & SD & Mean & SD & & \\
\hline Amplitude of initial VMO & 78.17 & 43.72 & 65.97 & 64.56 & 0.198 & Not Significant \\
Amplitude of initial VL & 120.45 & 47.26 & 78.31 & 40.55 & 0.014 & Significant \\
Amplitude of end VMO & 112.33 & 42.03 & 101 & 60.55 & 0.506 & Not Significant \\
Amplitude of end VL & 155.34 & 38.69 & 130.72 & 49.51 & 0.133 & Not Significant \\
$\begin{array}{c}\text { Amplitude ratio of initial } \\
\text { VMO/VL }\end{array}$ & 0.64 & 0.22 & 0.59 & 0.28 & 0.637 & Not Significant \\
$\begin{array}{c}\text { Amplitude ratio of end } \\
\text { VMO/VL }\end{array}$ & 0.7 & 0.21 & 0.60 & 0.21 & 0.034 & Significant \\
\hline
\end{tabular}

Table 5 shows the results analysis of exercise result differences seen in both exercise training group PRE method with and without EMG biofeedback monitoring. The above data showed that from the mean amplitude of the initial VMO, amplitude of initial VL, amplitude of end VL and amplitude ratio of the initial $\mathrm{VMO} / \mathrm{VL}$, no significant differences between these two groups with $p>0.05$. At the analysis of end VMO and amplitude ratio of end VMO/ VL, there was significant difference between the two groups $(p=0.014$ and $p=0.034)$.

\section{DISCUSSIONS}

This study was a clinical trial to obtain differences in the amplitude ratio of VMO and VL muscles before and after muscle strengthening exercises with PRE method with and without EMG biofeedback monitoring in patients with knee osteoarthritis at RSUD Dr. Soetomo.

Table 3 shows the results analysis of the mean amplitude of muscle contraction VMO, $\mathrm{VL}$ and amplitude ratio of $\mathrm{VMO} / \mathrm{VL}$ muscles before and after exercise in the PRE exercise 
group with EMG biofeedback monitoring. The mean amplitude of VMO and VL muscles increased significantly after exercise. The mean amplitude ratio of $\mathrm{VMO} / \mathrm{VL}$ muscles was not significantly increased after exercise. Table 4 shows the results analysis of the mean amplitude of VMO, VL muscles as well as the amplitude ratio of $\mathrm{VMO} / \mathrm{VL}$ muscles before and after exercise in the PRE exercise group without EMG biofeedback monitoring. The mean amplitude of VMO and VL muscles and the amplitude ratio of $\mathrm{VMO} / \mathrm{VL}$ muscles before and after exercise did not significantly increase post-exercise.

These results are consistent with the study of Dursun et $\mathrm{al}^{10}$ who showed an increase in the mean contraction rates of vastus medialis and vastus lateralis muscles in patellofemoral pain syndrome patients after 4 weeks of exercise with EMG biofeedback which is better than the group of patients who underwent conventional exercise. Table 5 shows the results analysis of differences in the exercise results of the two exercise groups. Statistical analysis showed that there was no significant difference of the amplitude of initial VMO, the amplitude of initial VL, the amplitude of end VL and the initial ratio amplitude between the groups using EMG biofeedback monitoring and without EMG biofeedback monitoring ( $p>$ $0.05)$. There were significant differences of the amplitude of end VMO and in the amplitude ratio of end $\mathrm{VMO} / \mathrm{VL}$ between the groups with and without EMG biofeedback monitoring $(p=0.014$ and $p=0.034)$. The results are consistent with previous studies by Krebs who evaluate differences of exercise with EMG biofeedback and conventional exercise in patients with post-meniscectomy knees. The study showed a better recovery in quadriceps in EMG biofeedback group compared with the conventional exercise group. The study of Ingerrsol et a ${ }^{10}$ also showed that exercise with EMG biofeedback is more superior than the strengthening exercise with PRE technique. In healthy people, according to Lucca and Recchiuti, exercise with EMG biofeedback produces a higher peak torque of quadriceps muscle than the exercise without EMG biofeedback. The study of Zhang Qi et al 11 showed similar results with this study, in which the ratio of $\mathrm{VMO} / \mathrm{VL}$ increased significantly in exercise with EMG biofeedback.

The amplitude of end VMO and the ratio of $\mathrm{VMO} / \mathrm{VL}$ at the end of the exercise PRE method with EMG biofeedback monitoring increased significantly when compared with the one without EMG biofeedback monitoring. This can be explained that EMG biofeedback is a medium that provides feedback in the form of audio-visual signal, so that training with EMG biofeedback is an effective therapeutic monitoring.

Amplitude of VMO, VL muscles and the amplitude ratio $\mathrm{VMO} / \mathrm{VL}$ muscles at the end of the PRE methods exercise without EMG biofeedback monitoring did not increase significantly. This was probably caused by the difference in the way of training and by the presence of subject activity as a confounding factor that cannot be controlled. The differences were due to different ways of training where the therapist involved as a personnel in this study and could also be due to not fully implemented exercise procedures which have been determined.

In patients with knee osteoarthritis, quadriceps muscle dysfunction can occur in the form of the VMO muscle weakness and tightness of the VL muscle. Moreover, patients with knee OA may also have neuromuscular dysfunction between the VMO and VL. The normal firing pattern is that the VMO activity occurred earlier than the VL, whereas the opposite occurs in knee OA, thus affecting the orientation of the patella and causes pain when the quadriceps muscles contracts, the VMO plays a role to pull the patella medially in order to keep the patella at the femoral sulcus. The position of the patella is affected by the direction of pull by the "quadriceps mechanism" and by the attachment position of the patellar tendon on the tibial tuberosity. This relationship is depicted as a point of Q, where this angle can be increased so that the patella tends to be pulled more laterally. The ratio of normal EMG activity of VMO/VL muscles in patients with knee OA is less than 1:1 which shows the VL muscle strength is greater than VMO. ${ }^{12,13}$ 


\section{CONCLUSIONS}

From this study, we conclude that the exercise of PRE method with EMG biofeedback monitoring significantly increased the amplitude of VMO and VL after exercise, while the amplitude ratio of $\mathrm{VMO} / \mathrm{VL}$ was not significantly increased. Meanwhile, without EMG biofeedback monitoring there were no significantly improvements of VMO and VL and the amplitude ratio of $\mathrm{VMO} / \mathrm{VL}$ as well. There were no significant improvement of VMO and VL amplitude and amplitude ratio in unmonitored group after exercise. There were anatomically changes in knee osteoarthritis where the patella got moved laterally, also an neuromuscular dysfunction between VMO and VL caused the muscle strength alteration. This are explaining the maximal amplitude ratio of $\mathrm{VMO} / \mathrm{VL}$ muscle before and after exercise in the EMG biofeedback monitoring group and in the group without EMG biofeedback monitoring was less than one.

\section{REFERENCES}

1. Santoso B. Tatalaksana Rehabilitasi Medik Penderita OA. Surabaya : SMF Rehabilitasi Medik RSU Dr. Soetomo; 2002.

2. Neumann DA. Kinesiology of the Musculoskeletal System. Foundation to Physical Rehabilitation. St Louis : Mosby; 2002.

3. Reyes TM, Reyes OBL.Therapeutic exercise I.Manila: U.S.T. Printing office; 1979.

4. Schumacher HR, Klippel JH, Koopman WJ. Osteoarthritis : Epidemiology, Pathology, and Pathogenesis. In : Schumacher HR, Klippel JH, Koopman WJ. Primer on the Rheumatic Diseases. 10th ed. Atlanta : Arthritis Foundation;
1993. p.184-190.

5. Elyas E. Pendekatan Terapi Fisik pada Osteoarthritis. Prosiding Pertemuan Ilmiah Tahunan PERDOSRI; 2002; Jakarta, Indonesia; 2002.

6. Brandt KD. Diagnosis and Nonsurgical Management of Osteoatrhritis. 2nd ed. Caddo : Professional Communications Inc; 2000.

7. Kisner C, Cosby LA. Therapeutic Exercise Foundation and Technique. 5th ed. Philadelpia : F.A. Davis Company; 2007.

8. Basmajian JV. Biofeedback in Physical Medicine and Rehabilitation . In : DeLisa J. Physical Medicine \& Rehabilitation Principles and Practice. 4th ed. Philadelpia : Lippincot Williams-Wilkins; 2005. p. 272-82

9. Basmajian JV. Biofeedback in Therapetic Exercise. In : Basmajian JV. Therapeutic Exercise. 3rd ed. London : Williams\&Wilkins; 1981. p 220-7

10. Dursun N, Dursun E, Kilic Z. Electromyographic BiofeedbackControlled Exercise Versus Conservative Care for Patellofemoral Pain Syndrome. Arch Phys Med Rehabil. Dec 2001; 82: 1692-4.

11. Qi Z, Ng G. EMG Analysis of Vastus Medialis Obliquus/Vastus Lateralis Activities in Subjects with PFPS before and after a Home Exercise Program. J Phys Ther Sci. 2006; 19. :131-7

12. Myomed 134. Enraf Nonius manual book. Rotterdam, Nederland;2005

13. Chunlong L. Electromyographic Biofeedback as treatment for training the vastus medialis obliquus and vastus lateralis in patients with patellofemoral pain syndrome. Available from : hhtp:// kf.gzrehab.com/news/photo/file108.doc. 


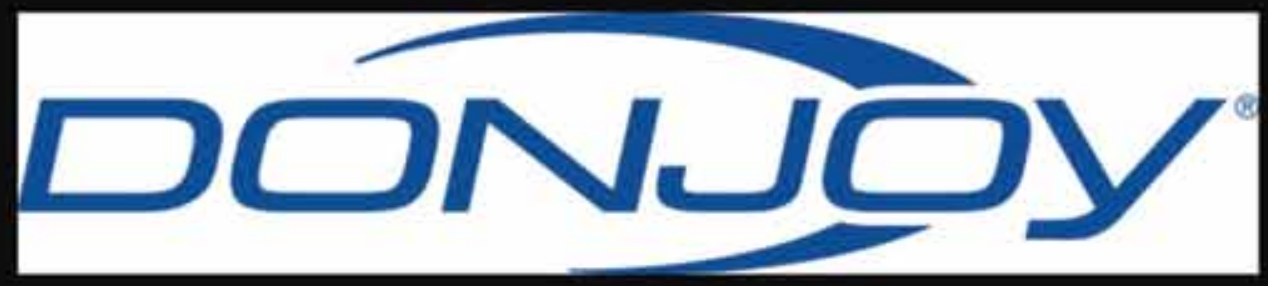

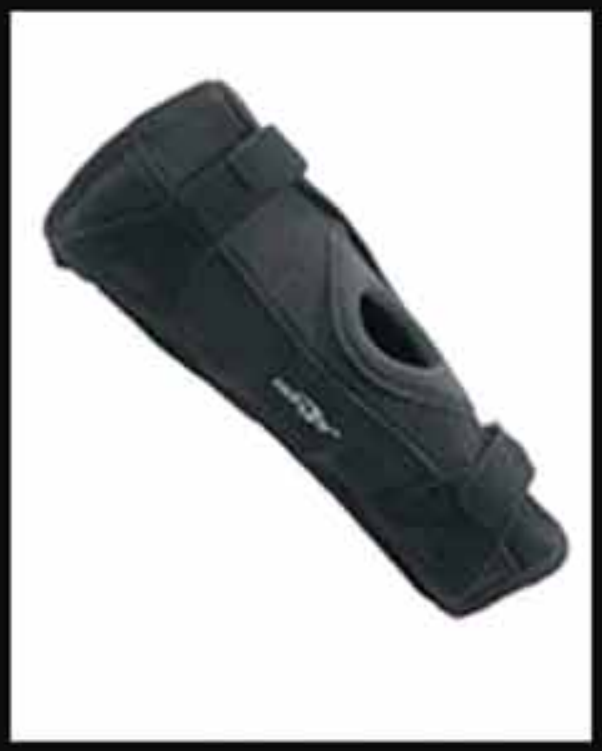

OA Lite

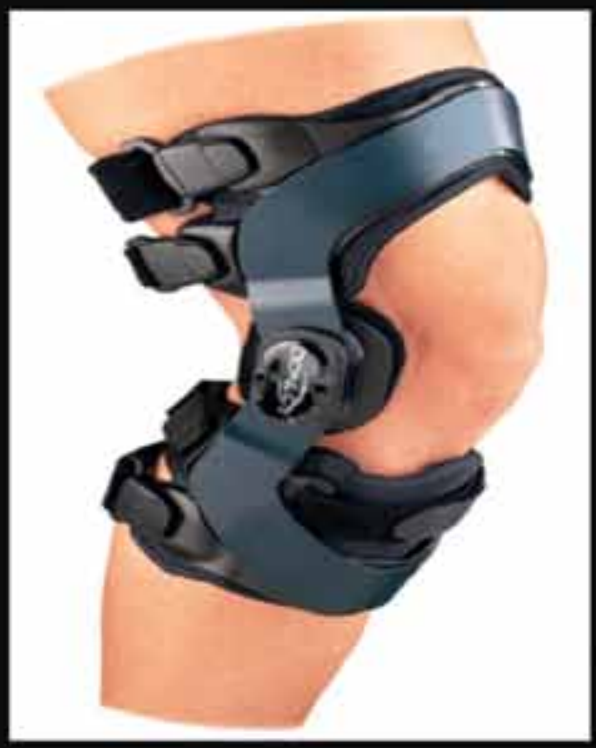

OA Everyday

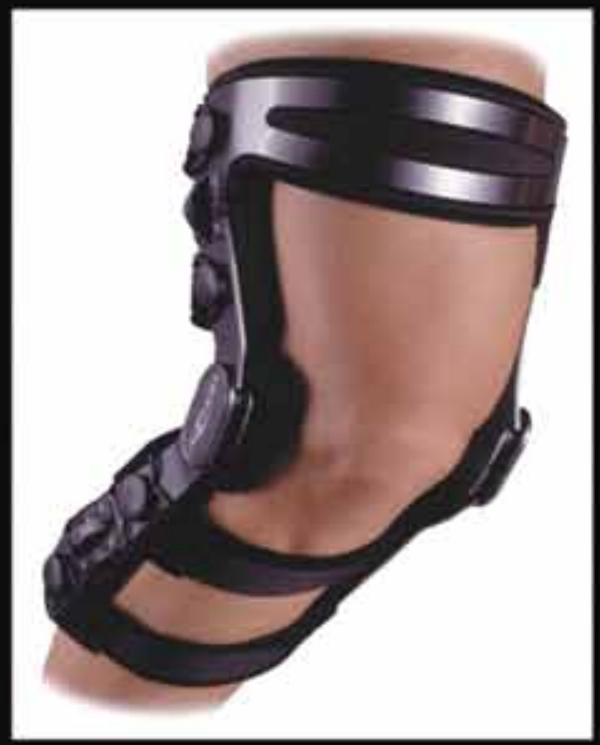

OA Adjuster (Mild OsteoArthritis) (Moderate OsteoArthritis) (Severe OsteoArthritis)

\section{"OA BRACE CORRECTION "}

Contact Person : Sulaeman Saleh ( 08138184 1840)

: Achmad Suud ( 08123026786 )

Kantor

Office

E-mail
:021-86903921

: PT. ENAM WARNA INDONESIA

: enam@indo.net.id

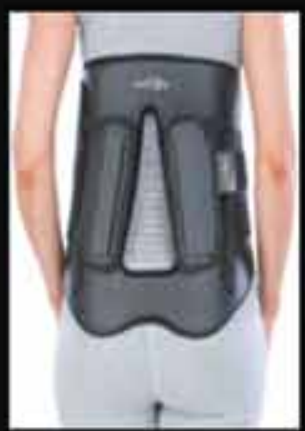

Donjoy Lite With ChairBack

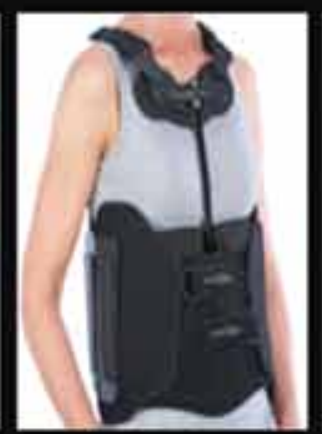

Donjoy Duel TLSO

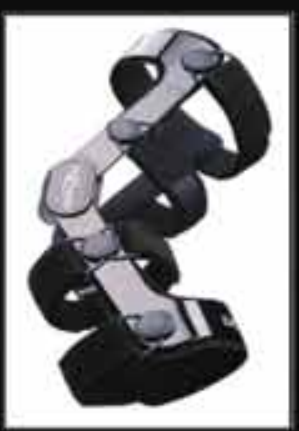

4 Titude

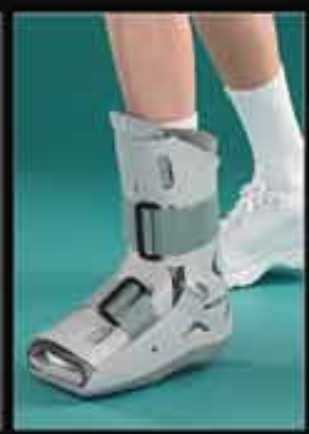

SP Walker

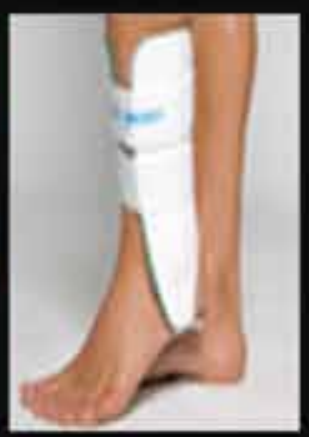

Air-Stirrup Ankle Brace

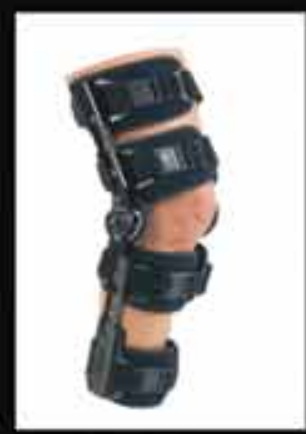

Cool Trom Advance 